\title{
Contextual Interference Effects in the Acquisition and Retention of Motor Tasks by Individuals With Mild Mental Handicaps
}

\author{
William J. Vincent \\ bill_vincent@byu.edu
}

Mary A. Painter

Kathleen B. Inman

Follow this and additional works at: https://scholarsarchive.byu.edu/facpub

Part of the Exercise Science Commons

\section{Original Publication Citation}

Contextual Interference Effects in the Acquisition and Retention of Motor Tasks by Individuals with Mild Mental Handicaps. Adapted Physical Activity Quarterly. Vol. 11, No. 4, October 1994. (M. Painter, K. Inman, W. J. Vincent).

\section{BYU ScholarsArchive Citation}

Vincent, William J.; Painter, Mary A.; and Inman, Kathleen B., "Contextual Interference Effects in the Acquisition and Retention of Motor Tasks by Individuals With Mild Mental Handicaps" (1994). Faculty Publications. 696.

https://scholarsarchive.byu.edu/facpub/696 accepted for inclusion in Faculty Publications by an authorized administrator of BYU ScholarsArchive. For more information, please contact ellen_amatangelo@byu.edu. 


\title{
Contextual Interference Effects in the Acquisition and Retention of Motor Tasks by Individuals With Mild Mental Handicaps
}

\author{
Mary A. Painter, Kathleen B. Inman, and William J. Vincent \\ California State University, Northridge
}

\begin{abstract}
The effects of contextual interference on motor skill acquisition and retention were examined in 24 subjects (mean age 13.9 years) with mild mental retardation and 24 chronologically age-matched subjects (mean age 13.11 years) with no disabilities. Subjects from each group were assigned randomly to either a blocked or a random practice schedule. All subjects performed 15 practice trials for each of three different beanbag throwing tasks, 45 trials total. Following a 10-min filled retention interval, 2 trials of each throw (6 total) were performed in a random order by all subjects. Accuracy scores were measured as absolute error from the target. The data revealed a significant interaction between ability groups and practice schedule. Post hoc analyses revealed that the retention scores of the mildly mentally handicapped subjects practicing under blocked conditions were significantly less accurate than scores of any of the other three acquisition groups. Significant effects in variable error retention scores indicated that subjects in the random practice condition performed more consistently than subjects in the blocked condition.
\end{abstract}

Finding effective and meaningful ways to facilitate learning in individuals with mental disabilities is a challenging endeavor. Teachers and practitioners can anticipate performance enhancement to a much greater extent than was once thought possible. Evidence indicates that instructional interventions designed to induce higher levels of cognitive processing have helped individuals with mental disabilities overcome strategy deficits (Campione, Brown, \& Ferrara, 1982; Hoover \& Wade, 1985). In particular, creating learning environments in which multiple contexts of related or similar skills are presented appears to facilitate learning, retention, and skill generalization (Brooks \& McCauley, 1984; Liberty, Haring, White, \& Billingsley, 1988).

Corresponding to the research which has demonstrated that individuals with mental disabilities can successfully discriminate cognitive tasks if presented

The authors are with the Department of Kinesiology, CSU, Northridge, 18111 Nordhoff, Northridge, CA 91330. 
with relational information is research with nondisabled individuals demonstrating that multiple contexts of within-task similarity lead to better retention and transfer. Known as contextual interference, this phenomenon was first reported in the verbal learning research by Battig $(1972,1979)$. Battig established that an increase in response similarity (high contextual interference) in turn increased task complexity and required higher levels of cognitive processing. The increased processing demands characteristically lead to poor performance during acquisition but facilitate positive transfer and retention.

Contextual interference effects have become a major focus of motor learning research in recent years (see Magill \& Hall, 1990). The manipulation of practice conditions such that successive practice trials are disrupted to a greater or lesser degree has been examined for impact upon the retention and transfer of motor tasks. Research paradigms on contextual interference effects in the motor domain traditionally have structured low contextual interference conditions by conducting all the trials of one task prior to introducing a new task (blocked practice). High contextual interference conditions are created by randomly ordering the practice of two or more tasks across acquisition trials (random practice).

Studies with nondisabled subjects have demonstrated the positive effects of high contextual interference on the retention and transfer of motor tasks (Del Rey, 1989; Del Rey, Whitehurst, Wughalter, \& Barnwell, 1983; Del Ray, Wughalter, \& Carnes, 1987; Goode \& Magill, 1986; Jelsma \& Van Merrienboer, 1989; Lee \& Magill, 1983; Lee, Magill, \& Weeks, 1985; Shea \& Morgan, 1979). There have been, however, investigations in which contextual interference effects have not been supported (Del Rey, Whitehurst, \& Wood, 1983; Dunham, 1977, 1978; Heitman \& Gilley, 1989; Whitehurst \& Del Rey, 1983). For an in-depth review of the early contextual interference research in the motor domain, see Magill and Hall (1990).

More recently, studies employing a contextual interference paradigm have used individuals with mental disabilities as the subjects. The results have been as equivocal as studies with nondisabled subjects. Edwards, Elliott, and Lee (1986) found a positive effect of high contextual interference on the transfer performance of an anticipation timing task with a Down syndrome group of adolescents and a mental age-matched (MA-matched) group of children, but their results were not statistically significant. Porretta (1988) also was unable to obtain statistical significance on the transfer and retention of a beanbag tossing task with mildly mentally handicapped ( $\mathrm{MMH})$ children; however, subjects in the random practice condition tended to demonstrate better performance. Del Rey and Stewart (1989) did obtain significant contextual interference effects with $\mathrm{MMH}$ subjects on the retention of an anticipation timing task, but these same subjects were unable to transfer their performance to a new speed. Heitman and Gilley (1989) were unable to demonstrate contextual interference effects using a pursuit rotor task with subjects who were trainable mentally retarded (TMR).

Porretta and O'Brien (1991) suggested that these marginal results may be due to an insufficient number of trials and/or practice sessions. They hypothesized that since individuals with mental impairments take longer to learn motor skills, additional practice sessions employing high contextual interference conditions (random practice) would result in better transfer and retention. The MMH subjects in their study practiced an anticipation timing task under a random, blocked, or serial schedule of 48 trials per day for 2 consecutive days. The subjects were 
transferred to a novel speed for 4 consecutive trials immediately following practice. They repeated this transfer task 2 days later. The results were significant for both the transfer data and the "transfer-retention" data. Subjects in the random practice condition performed with less absolute constant error than subjects in the blocked group. The performance of subjects in the serial group did not differ significantly from performances of either the random or blocked groups.

As a result of their study, Porretta and O'Brien suggested that the use of high contextual interference practice schedules with mentally impaired individuals is advantageous if practice time is sufficient for subjects to process the multiple contexts of the skills being learned. They surmised that future studies using children with mental disabilities as subjects should employ several practice sessions to elicit the contextual interference effect.

The in-depth review of the contextual interference literature by Magill and Hall (1990) suggests that the generalizability of contextual interference effects may depend upon the task variations that are practiced. Contextual interference effects were found when the task variations involved different motor programs, in other words when the tasks were distinguished by variations in their relative timing, sequence of events, and/or spatial configurations. Magill and Hall proposed that between-motor program variations create a more difficult learning context than do within-motor program variations. Restructuring invariant features of a task requires more dynamic information processing than does changing a task parameter such as force, duration, or the size of the spatial configuration. The greater processing demands inherent in performing tasks with different motor programs thus contribute to significant contextual interference situations. Although there has been more recent evidence to further support the Magill and Hall conclusion (Chamberlin, Rimer, \& Skaggs, 1990; Crumpton, AberdrothSmith, \& Chamberlin, 1990; Lee, Wulf, \& Schmidt, 1990; McNevin \& Magill, 1992; Wood \& Ging, 1991; Wulf \& Lee, 1992), there also has been some research contradicting this hypothesis (Sekiya, Magill, Sidaway, \& Anderson, 1992; Wulf, 1992).

None of the contextual interference studies using individuals with mental deficiencies as subjects have employed task variations with different motor programs. The variations reported have been context variations resulting from a change in absolute speed of task stimulus (Del Rey \& Stewart, 1989; Edwards et al., 1986; Heitman \& Gilley, 1989; Porretta \& O'Brien, 1991) or absolute weight of the object projected (Porretta, 1988) or from variations in the projection surface (Porretta, 1982). Although Porretta and O'Brien (1991) suggested that contextual interference studies using children with intellectual disabilities should be designed to include an increased number of practice trials presented on several occasions, perhaps practicing skills with different motor programs would produce contextual interference effects within a single practice session.

The purpose of this study was to investigate contextual interference effects on motor skill retention in individuals with mild mental retardation using context variations that resulted from tasks with different motor programs. Studies with nondisabled subjects have demonstrated results in a single acquisition session (Gabriele, Hall, \& Buckolz, 1987; Jelsma \& Merrienboer, 1989; Lee \& Magill, 1983; Shea \& Morgan, 1979); therefore, skill acquisition was limited to a single session in the present study. This seemed like an appropriate first step in differentiating between motor programming considerations and multiple practice sessions. The 
motor program variations were evidenced in the different timing and spatial configurations inherent in three distinct throwing patterns performed by the subjects.

\section{Method}

\section{Subjects}

The subjects included 24 students enrolled in $\mathrm{MMH}$ classrooms at a public junior high school and senior high school located in a suburb of Los Angeles County. Although socioeconomic status was not directly assessed, the students served by these schools appeared to come from families of middle to low-middle socioeconomic status. In compliance with recent private acts, the school district did not provide specific IQs. Assignment to the MMH classroom, however, was based on IQ scores ranging from 50 to 80 . Physical education instruction for these students occurred in an adapted physical education setting in which classmates demonstrated a mixture of mental, physical, and learning disabilities ranging from mild to severe.

The ages of the $\mathrm{MMH}$ subjects ranged from 11 to 18 years, with a mean age of 13.9. The large age range in this sample reflected the extent of $\mathrm{MMH}$ students among the approximately 5,000 students enrolled in the junior and senior high schools from which permission to conduct the study was obtained. Twentyfour gender-matched and chronologically age-matched (CA-matched) students (mean age 13.11 years) from the same secondary school district also were included as subjects in the study. All subjects were volunteers for whom signed consent was obtained. None of the subjects had concomitant sensory or physical impairments. All subjects were randomly assigned to training groups such that an equal number of males and females were represented in each training group.

\section{Procedure}

The tasks performed in this study involved throwing beanbags from a distance of $20 \mathrm{ft}(6.10 \mathrm{~m})$ to a fixed target. The beanbags were made of cotton fabric, measured $8 \mathrm{~cm} \times 8 \mathrm{~cm}$, and weighed $5 \mathrm{oz}$. Three different throwing patterns were performed by the subjects: an underhand throw, an overhand throw, and a hook throw. The hook throw was performed with the body positioned sideways to the target and the throwing arm away from the target. The arm was abducted laterally such that the beanbag was projected up and over the head. The timing and spatial configurations of the three throwing patterns were clearly different enough from one another to suggest that they represented three distinct motor programs. All throws were performed with the preferred hand.

When throwing patterns are employed in sports and games, the task objective typically involves accuracy in throwing to either a stationary or a moving target at least $3 \mathrm{ft}(0.91 \mathrm{~m})$ off the ground. In this study the target was a 12-in.square $(0.30 \mathrm{~m})$ softball base located at floor level; thus, typical throwing patterns were viewed by the researchers as being performed in a novel environmental context for all students in the study. Absolute error from the target was measured in centimeters.

Both the $\mathrm{MMH}$ and nondisabled subjects were randomly assigned to either a blocked practice schedule or a random practice schedule. Every subject per- 
formed 15 practice trials of the underhand throw, 15 practice trials of the overhand throw, and 15 practice trials of the hook throw. Subjects in the blocked practice group performed all trials of one skill before practicing the next skill. Presentation of the throws was counterbalanced across subjects. The subjects in the random practice group practiced all trials of each skill in a random order, with no skill performed more than two times consecutively. Following a 10-min filled retention interval in which the subjects played volleytennis, 2 trials of each throw (6 trials) were again performed. The retention trials were performed in a random order by all subjects.

The motor tasks were administered to each subject individually by two examiners. Each throw was demonstrated twice by one of the test administrators. One administrator stood with the subject and was responsible for ensuring that the subject was behind the throwing line. The second administrator informed the subject of the type of throw to be performed, immediately marked all throws at their first point of contact with the ground, and then measured the distance between this point and the closest edge of the target. The testing took place over a period of 3 weeks, with individual sessions lasting $10 \mathrm{~min}$.

Due to the distractibility of the $\mathrm{MMH}$ population, specific predetermined verbal encouragement such as "good shot" and "nice job" was given after each practice and retention trial by the second administrator to both the $\mathrm{MMH}$ and the nondisabled participants. No verbal knowledge of results was given in either acquisition or retention, but performance results were directly observable by the subjects.

\section{Statistical Analysis}

Absolute error and variable error served as the dependent measures of performance. The 45 practice trials were grouped for analysis into 15 practice blocks of 3 trials each. The mean absolute error score for each block of data was used in the analysis, as well as the variable error score for each block. Absolute error scores ignore the direction of the deviation score. Since the direction of the error was not the focus of this study, absolute error scores were regarded as the most appropriate measure of accuracy (Pigott \& Shapiro, 1984; Porretta, 1988). Variable error estimates the variability of the scores around the mean response and thereby served as a measure of performance consistency.

The practice data were subjected to a 2 (Group: MMH, CA-matched) $\times 2$ (Schedule: random, blocked) $\times 15$ (Blocks of Practice Trials) ANOVA with repeated measures on the last factor. The Greenhouse-Geisser correction factor was employed when the assumption of sphericity was not met. A 2 (Group) $\times$ 2 (Schedule) ANOVA was conducted on the retention data. One additional repeated measures ANOVA was conducted to compare the data on the last trial block (Trial Block 15) with the retention data. For all analyses, Tukey's HSD test was employed to determine significant differences between main effects. The accepted level of significance was set at the .05 confidence level.

\section{Results}

\section{Absolute Error}

Analysis of the practice data revealed significant main effects for groups, $F(1,44)=$ $12.45, p<.001$, and trial blocks, $F(14,616)=8.24, p=.001$. The CA-matched 
subjects performed the beanbag throws with less absolute error (greater accuracy) than the MMH subjects. Performance improved significantly from Trial Block 1 to Trial Block 15. However, a significant trial block by schedule interaction, $F(14$, $616)=3.84, p<.001$, indicated that the groups practicing under blocked conditions did not experience the same pattern in performance improvement across the trial blocks as experienced by the groups practicing under random conditions. On Trial Blocks 6 and 11 a relatively large regression in performance was demonstrated by both the $\mathrm{MMH}$ and the CA-matched subjects experiencing blocked acquisition schedules. The groups experiencing random practice did not demonstrate this same regression. This regression most likely was caused by the introduction of a different throwing task on Trials 16 and 33, when a new block of acquisition trials was begun.

Tukey's post hoc analyses of the trial-block scores for practice schedules revealed that a significant reduction in absolute error $(p<.05)$ occurred between Block 1 and Block 15 for the groups experiencing random practice, but not for the groups experiencing blocked practice. Although a three-way interaction (block by schedule by group) was not significant, the improvement in the scores of the $\mathrm{MMH}$ subjects experiencing random practice is particularly noteworthy (see Figure 1).

Analysis of the absolute error retention data disclosed significant main effects for both groups, $F(1,44)=8.15, p<.007$, and practice schedules, $F(1$, $44)=15.77, p<.001$, as well as a significant interaction between these two factors, $F(1,44)=6.02, p<.02$. Post hoc analysis revealed that the retention scores of the $\mathrm{MMH}$ subjects practicing under blocked conditions were significantly less
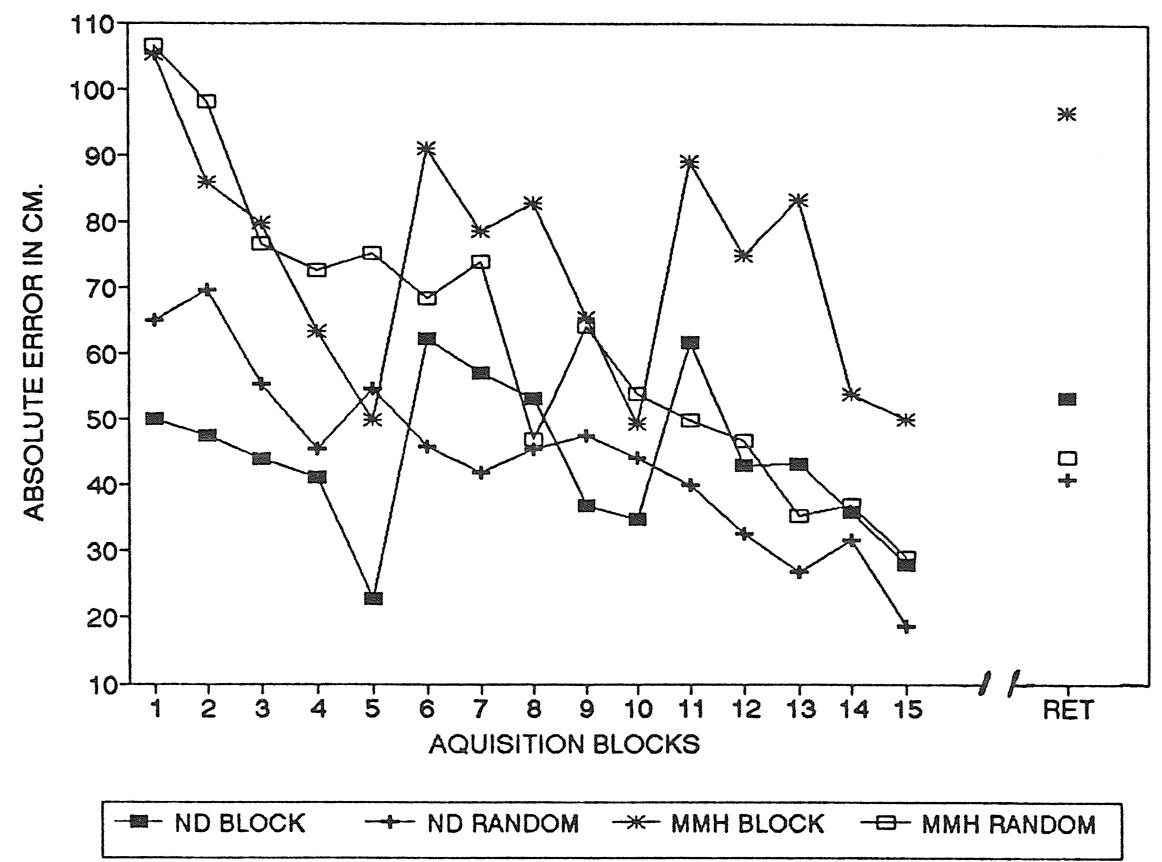

Figure 1 - Mean absolute error for acquisition and retention trial blocks. ND = nondisabled; $\mathrm{MMH}$ = mildly mentally handicapped. 
accurate $(p<.05)$ than any of the other three acquisition groups. There were no other significant differences in the retention data.

A significant trial block, $F(1,44)=56.99, p=.001$, schedule, $F(1,44)=$ $12.19, p<.01$, and block by schedule interaction, $F(1,44)=5.62, p<.02$, was obtained in the analysis comparing the last trial block with the retention data. Although an increase in absolute error occurred in the retention data for both the random and blocked schedules, the groups practicing under a blocked schedule demonstrated a significantly greater increase than the groups practicing under a random schedule.

\section{Variable Error}

No significant differences in variable error scores were found between either practice schedules or groups. These results indicate that the scores of the subjects experiencing blocked practice conditions were just as consistent as the scores of the subjects practicing under random conditions. Additionally, the lack of significant difference in variable error between the CA-matched subjects and the MMH subjects implies that the consistency of performance was similar for both groups of subjects (see Figure 2).

A significant trial block, $F(14,616)=7.32, p=.001$, and block by schedule interaction, $F(14,616)=2.22, p<.006$, indicated that while variable error was reduced from Trial Block 1 to Trial Block 15, this improvement in performance consistency was not the same for the blocked and the random schedules. The

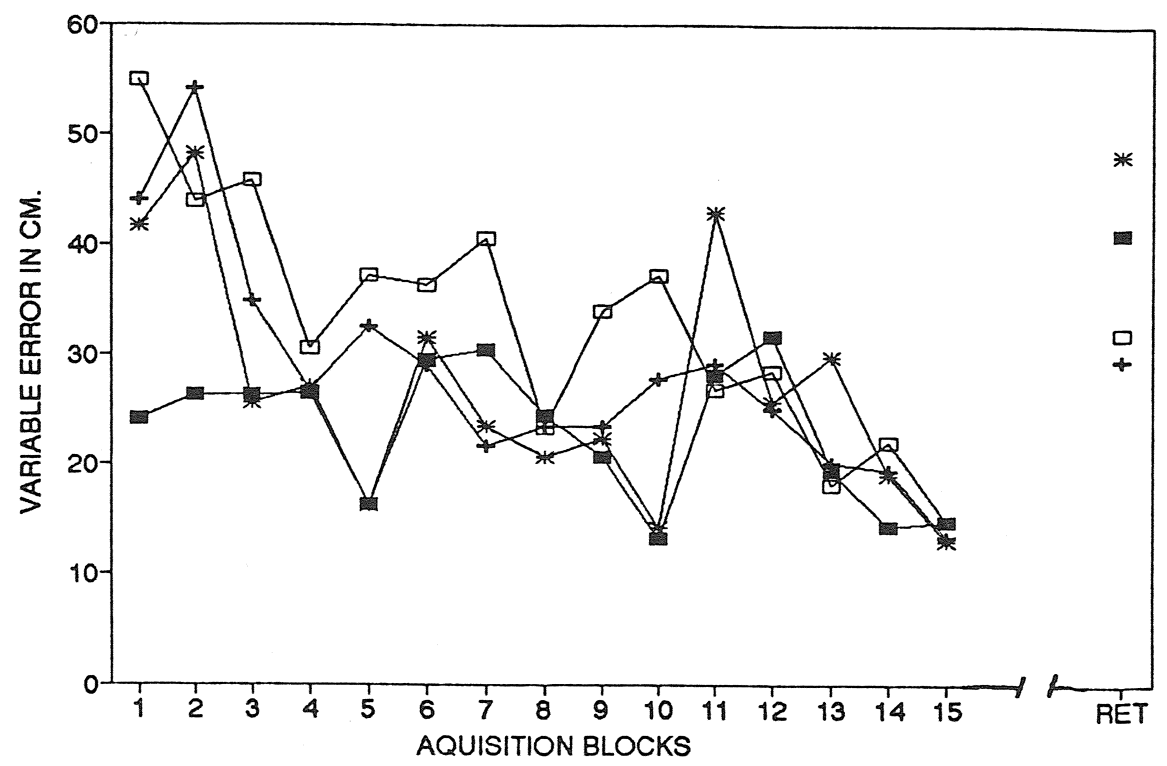

$\square-$ MMH RANDOM

Figure 2 - Mean variable error for acquisition and retention trial blocks. ND = nondisabled; $M M H$ = mildly mentally handicapped. 
results of post hoc analyses of variable error were similar to those for absolute error. Significant improvement was seen in the scores of groups practicing under random conditions, but not in the scores of the groups practicing under blocked conditions.

The only significant difference found in the variable error scores during retention was between practice schedules, $F(1,44)=7.94, p<.007$. Subjects in the random practice groups performed more consistently than subjects in the blocked practice groups. There were no other significant differences in the retention data.

Similar to the absolute error scores, however, there was a significant block, $F(1,44)=76.13, p=.001$, schedule, $F(1,44)=6.54, p<.01$, and block by schedule interaction, $F(1,44)=6.62, p<.01$, in the analysis comparing the last trial block with the retention data. Both the random and the blocked schedules revealed a significant increase in variable error during retention, but the groups practicing under a blocked schedule demonstrated a significantly greater increase than the groups practicing under a random schedule.

\section{Discussion}

Consistent with studies that have compared the motor performances of individuals with mental disabilities to CA-matched subjects, this study showed that nondisabled subjects demonstrated better accuracy overall than the MMH subjects in both acquisition and retention (Dobbins \& Rarick, 1976; Francis \& Rarick, 1959; Porretta, 1982; Rarick, Widdop, \& Broadhead, 1970). The trend in the mean scores across blocks shows that these differences were accentuated early in practice but were reduced considerably at the end of practice (see Figure 1). Indeed, the post hoc analyses revealed no significant differences between groups by the end of the acquisition trials.

The results of this study suggest that, given sufficient practice, $\mathrm{MMH}$ students can acquire accuracy and consistency in throwing tasks similar to that demonstrated by nondisabled students. This performance equivalency is not retained to the same degree, however. Although all groups experienced a significant increase in absolute error between Trial Block 15 and the retention trials, significant differences between groups in the retention data indicated that the scores obtained by the nondisabled subjects as a group were again more accurate than those obtained by the $\mathrm{MMH}$ subjects.

The significantly better acquisition performance of the groups practicing under random conditions is contrary to Battig's $(1972,1979)$ theory of contextual interference, which suggests that subjects performing under random conditions should perform worse during the acquisition of a skill. It is possible that the results reflect the large variability evidenced in the absolute error and variable error scores by both the MMH subjects and the nondisabled subjects. Despite this theoretical discrepancy in the acquisition phase, trends in the data provide some support for a contextual interference effect in the motor skill retention of individuals with mild mental disabilities.

In contrast to most of the contextual interference research in which individuals with mental disabilities were subjects, this study showed that the $\mathrm{MMH}$ random practice group performed with significantly better accuracy during retention than the $\mathrm{MMH}$ blocked group. Blocked practice of throwing tasks with different motor 
programs does not appear to be conducive to random retention in $\mathrm{MMH}$ subjects. These results could reflect the differences existing between the processing activities experienced by the blocked group in the practice conditions and the processing activities required during the retention trials. Subjects in the random practice group did not change their performance conditions between acquisition and retention, whereas subjects in the blocked practice group were performing randomly for the first time. Interpretation of the retention data is limited by the absence of blocked retention trials, but the results do reveal an advantage of random practice to random retention for the $\mathrm{MMH}$ subjects in this study.

Processing differences between acquisition and retention do not explain the absolute error retention scores of the nondisabled students who practiced under blocked schedules. There were no significant differences between the retention scores of the nondisabled subjects practicing under blocked conditions and those practicing under random conditions, although it should be noted that the absolute error for the subjects experiencing random practice conditions was less. It is possible that the beanbag tossing activity was not developmentally appropriate for the nondisabled subjects. Perhaps these students already were fairly proficient at the tasks and any improvement evidenced during acquisition was due to skill refinement, not new learning. If this were the case, retention differences between the groups would be minimal and, indeed, they were.

Random retention was chosen for this study because most game situations in physical education classes call for random responses. The lesson plan format for many physical education classes often entails a practice session followed by some type of lead-up or actual game play. In open game situations continual repetition of the same motor skill is not conducive to effective performance.

Not only are random responses essential to game play, but novel responses also enhance performance when unexpected situations arise. The ability to perform a motor task in a novel situation following acquisition of the task is usually tested with a transfer task. A limitation in the design of this study is the lack of a test for transfer effects to a novel task; therefore, this study does not fully investigate the contextual interference phenomenon. As such, the findings should be considered preliminary and the results should be interpreted with caution. Nevertheless, the results suggest that random practice conditions in adapted physical education classes might facilitate the performance of MMH students in open game situations calling for random responses. Further research is needed to confirm this hypothesis.

Elaboration theory (Shea \& Zimny, 1983) and action plan reconstruction theory (Lee \& Magill, 1983) have both been offered as explanations for contextual interference effects. Elaboration theory suggests that the processing of multiple strategies in a varied context during practice results in the elaboration of memory representations, which leads to enhanced retention and transfer. The more extensive multiple and varied processing demanded in random practice conditions results in poor performance during acquisition but enhanced retention and transfer. Action plan reconstruction theory proposes that it is the accessibility of the variable strategies (or action plans), not the multiple processing of them, which causes active reconstruction of memory representations. Since blocked conditions would not require action plan reconstruction trial to trial, acquisition performance would be better for subjects experiencing blocked schedules than for subjects exposed to random practice conditions. 
Porretta and O'Brien (1991) suggested that the lack of performance differences between MMH subjects during acquisition may be the result of similarities in how the random and blocked learning groups construct action plans. Because of their mental deficit, all MMH subjects may need to engage in active processing when learning motor tasks regardless of the practice conditions imposed. In contrast, nondisabled subjects may need to engage in active processing only in random learning conditions. The lack of a three-way interaction (block by schedule by group) in this study fails to distinguish between the specific learning groups and, consequently, challenges this processing argument.

Porretta and O'Brien also pointed out that a reduction in group differences across acquisition trials is consistent with the elaboration view of contextual interference. Indeed, Shea and Zimny (1983) suggested that a limit may be reached in which the elaboration of cognitive processing for a simple task can be performed. Acquisition and retention differences may not have been evidenced between the nondisabled subjects because the tasks already were well learned and further elaboration was simply a refinement of the memorial representation of the tasks.

In contrast, the absolute error scores were much greater for the $\mathrm{MMH}$ subjects than for the nondisabled subjects on Block 1. By Block 15 these differences were reduced considerably. Perhaps in the early acquisition trials all the MMH subjects required more extensive elaboration and distinctive processing for the different throws than did the nondisabled subjects, but as practice continued the performance differences were reduced and the amount of elaborative processing between the groups became similar. At the same time, the task may have been novel enough for the MMH subjects that contextual interference effects became apparent during retention when the random acquisition group demonstrated significantly better performance than the blocked $\mathrm{MMH}$ group.

While the processes underlying the contextual interference phenomenon in both nondisabled individuals and individuals with mental disabilities merit further investigation, it is the impact of the learning context on retention and transfer that is the critical issue for the practitioner (Lee \& Magill, 1983). The question to be answered is whether varied, high-interference (random) practice schedules are more effective than low-interference (blocked) schedules in the retention and transfer of a motor skill. The distinguishing feature in this study rests in the positive contextual interference effects for retention found within a single practice session in subjects with mild mental handicaps. The results in this study are partially consistent with previous contextual interference studies in which subjects without disabilities practiced tasks with different motor programs (Chamberlin et al., 1990; Crumpton et al., 1990; Lee et al., 1990; McNevin \& Magill, 1992; Wood \& Ging, 1991; Wulf \& Lee, 1992).

Porretta and O'Brien (1991) suggested that because individuals with mental disabilities take longer to learn motor skills, practice needs to be distributed across sessions for these subjects before contextual interference effects can be found. Although the results of the current study do not provide support for the Porretta and O'Brien hypothesis, they do not refute it either. The limitations of this study are noted and the results must be considered preliminary. In order to distinguish between the motor program variable and the number of practice sessions, further research that examines the impact of contextual interference in individuals with mental disabilities when tasks represent different motor programs is needed. Future 
studies should incorporate (a) a balanced design for both random and blocked retention, (b) a transfer task, and (c) a retention interval of 1 day or more.

\section{References}

Battig, W.F. (1972). Intratask interference as a source of facilitation in transfer and retention. In R.F. Thompson \& J.F. Voss (Eds.), Topics in learning and performance (pp. 131-159). New York: Academic Press.

Battig, W.F. (1979). The flexibility of human memory. In L.S. Cermak \& F.I.M. Craik (Eds.), Levels of processing and human memory (pp. 23-44). Hillsdale, NJ: Erlbaum.

Brooks, P.H., \& McCauley, C. (1984). Cognitive research in mental retardation. American Journal of Mental Deficiency, 88, 479-486.

Campione, J.D., Brown, A.L., \& Ferrara, R.A. (1982). Mental retardation and intelligence. In R.J. Sternberg (Ed.), Handbook of human intelligence (pp. 392-490). New York: Cambridge University Press.

Chamberlin, C.J., Rimer, T.N., \& Skaggs, D.J. (1990). The ecological validity of the contextual interference effect: A practical application to learning the jump shot in basketball. Proceedings of the North American Society for the Psychology of Sport and Physical Activity (p. 67). Houston, TX: NASPSPA.

Crumpton, R.L., Aberdroth-Smith, J., \& Chamberlin, C.J. (1990). Contextual interference and the acquisition of motor skills in a field setting. Proceedings of the North American Society for the Psychology of Sport and Physical Activity (p. 58). Houston, TX: NASPSPA.

Del Rey, P. (1989). Training and contextual interference effects on memory and transfer. Research Quarterly for Exercise and Sport, 60, 342-347.

Del Rey, P., \& Stewart, D. (1989). Organizing input for mentally retarded subjects to enhance memory and transfer. Adapted Physical Activity Quarterly, 6, 247-254.

Del Rey, P., Whitehurst, M., \& Wood, J. (1983). Effects of experience and contextual interference on learning and transfer. Perceptual and Motor Skills, 56, 581-582.

Del Rey, P., Whitehurst, M., Wughalter, E., \& Barnwell, J. (1983). Contextual interference and experience in acquisition and transfer. Perceptual and Motor Skills, 57, 241242.

Del Rey, P., Wughalter, E., \& Carnes, M. (1987). Level of expertise, interpolated activity, and contextual interference effects on memory and transfer. Perceptual and Motor Skills, 64, 275-284.

Dobbins, D.A., \& Rarick, G.L. (1976). Separation potential of educable retarded and intellectually normal boys as a function of motor performance. Research Quarterly, 47, 346-356.

Dunham, P. (1977). Effect of practice order on the efficiency of bilateral skill acquisition. Research Quarterly, 48, 284-287.

Dunham, P. (1978). Retention of bilateral performance as a function of practice order. Perceptual and Motor Skills, 46, 43-46.

Edwards, J.M., Elliott, D., \& Lee, T.D. (1986). Contextual interference effects during skill acquisition and transfer in Down's Syndrome adolescents. Adapted Physical Activity Quarterly, 3, 250-258.

Francis, R.J., \& Rarick, G.L. (1959). Motor characteristics of the mentally retarded. American Journal of Mental Deficiency, 63, 792-811.

Gabriele, T.E., Hall, C.R., \& Buckolz, E.E. (1987). Practice schedule effects on the acquisition and retention of a motor skill. Human Movement Science, 6, 1-16. 
Goode, S.L., \& Magill, R.A. (1986). Contextual interference effects in learning three badminton serves. Research Quarterly for Exercise and Sport, 57, 308-314.

Heitman, R.J., \& Gilley, W.F. (1989). Effects of blocked versus random practice by mentally retarded subjects on learning a novel skill. Perceptual and Motor Skills, 69, 443-447.

Hoover, J.H., \& Wade, M.G. (1985). Motor learning theory and mentally retarded individuals: A historical review. Adapted Physical Activity Quarterly, 2, 228-252.

Jelsma, O., \& Van Merrienboer, J.J.G. (1989). Contextual interference with reflectionimpulsivity. Perceptual and Motor Skills, 68, 1055-1064.

Liberty, K.A., Haring, N.G., White, O.R., \& Billingsley, F. (1988). A technology for the future: Decision rules for generalization. Education and Training in Mental Retardation, 23, 315-325.

Lee, T.D., \& Magill, R.A. (1983). The locus of contextual interference in motor-skill acquisition. Journal of Experimental Psychology: Learning, Memory, and Cognition, 9, 730-746.

Lee, T.D., Magill, R.A., \& Weeks, D.J. (1985). Influence of practice schedule on testing schema theory predictions in adults. Journal of Motor Behavior, 17, 282-289.

Lee, T.D., Wulf, G., \& Schmidt, R.A. (1990). Action plan reconstruction and the contextual interference effect on motor learning. Proceedings of the North American Society for the Psychology of Sport and Physical Activity (p. 10). Houston, TX: NASPSPA.

Magill, R.A., \& Hall, K.G. (1990). A review of the contextual interference effect in motor skill acquisition. Human Movement Science, 9, 241-289.

McNevin, N.H., \& Magill, R.A. (1992). A task dynamics approach to the contextual interference effect. Proceedings of the North American Society for the Psychology of Sport and Physical Activity (p. 106). Pittsburgh, PA: NASPSPA.

Pigott, R.E., \& Shapiro, D.C. (1984). Motor schema: The structure of the variability session. Research Quarterly for Exercise and Sport, 55, 41-45.

Porretta, D.L. (1982). Motor schema formation by EMR boys. American Journal of Mental Deficiency, 87, 154-172.

Porretta, D.L. (1988). Contextual interference effects on the transfer and retention of a gross motor skill by mildly mentally handicapped children. Adapted Physical Activity Quarterly, 5, 332-339.

Porretta, D.L., \& O’Brien, K. (1991). The use of contextual interference trials by mildly mentally handicapped children. Research Quarterly for Exercise and Sport, 62, 244-248.

Rarick, G.L., Widdop, J.H., \& Broadhead, G.D. (1970). The physical fitness and motor performance of educable mentally retarded children. Exceptional Children, 36, 509519.

Sekiya, H., Magill, R.A., Sidaway, B., \& Anderson, D. (1992). The contextual interference effect with same and different motor programs. Proceedings of the North American Society for the Psychology of Sport and Physical Activity (p. 116). Pittsburgh, PA: NASPSPA.

Shea, J.B., \& Morgan, R.L. (1979). Contextual interference effects on the acquisition, retention, and transfer of a motor skill. Journal of Experimental Psychology: Human Learning and Memory, 5, 179-187.

Shea, J.B., \& Zimny, S.T. (1983). Context effects in memory and learning movement information. In R.A. Magill (Ed.), Memory and control of action (pp. 345-366). Amsterdam: North-Holland. 
Wood, C.A., \& Ging, C.A. (1991). The role of interference and task similarity on the acquisition, retention, and transfer of simple motor skills. Research Quarterly for Exercise and Sport, 62, 18-26.

Whitehurst, M., \& Del Rey, P. (1983). Effects of contextual interference, task difficulty, and levels of processing on pursuit tracking. Perceptual and Motor Skills, 57, 619628.

Wulf, G. (1992). Contextual interference and reduced KR frequency enhance the learning of generalized motor programs. Proceedings of the North American Society for the Psychology of Sport and Physical Activity (p. 127). Pittsburgh, PA: NASPSPA.

Wulf, G., \& Lee, T.D. (1992). Contextual interference in movements of the same class: Differential effects on program and parameter learning. Proceedings of the North American Society for Psychology of Sport and Physical Activity (p. 126). Pittsburgh, PA: NASPSPA. 